\title{
Coqblin-Schrieffer impurity in a singular metal
}

\author{
Yupeng Wang \\ Department of Physics, Florida State University, Tallahassee, FL 32306, USA, \\ and Institute of Physics, Chinese Academy of Sciences, Beijing 100080, \\ People's Republic of China \\ P. Schlottmann \\ Department of Physics, Florida State University, Tallahassee, FL 32306, USA
}

\begin{abstract}
A Coqblin-Schrieffer impurity of spin $S$ coupled to the boundary of an open SU(N)-invariant $t-J$ chain with $N=2 S+2$ is studied. The model is integrable as a function of one coupling parameter $v$ for arbitrary spin and band filling. The system is coupled to a reservoir of electrons and as a function of the chemical potential the host chain has a critical point corresponding to the van Hove singularity of the empty band of charges. In the neighborhood of this critical point we study the low temperature properties of the impurity as a function of $v$. For constant chemical potential we obtain two critical coupling values $v_{1}=-1 / 2$ and $v_{2}=0$ separating phases in which the impurity spin is completely compensated (Kondo effect), partially screened (undercompensated impurity) or decoupled from the chain (free spin). The partial compensation occurs as a consequence of a thermally activated Kondo effect in the region $v_{1}<v<v_{2}$. The possible realization of such a finite temperature Kondo effect in narrow-gap semiconductors is discussed.
\end{abstract}

75.20.Hr, 72.15.Qm

The unusual non-Fermi-liquid properties observed in several heavy-fermion compounds [1] are frequently attributed to the existence of a quantum critical point (QCP). For instance, Kondo impurities in a multi-channel electron host [2] provide such a QCP in the overscreened case with non-Fermi-liquid behavior in a variety of low-temperature thermodynamic quantities [3]. Magnetic impurities coupled to a Luttinger liquid may behave rather differently from those in a Fermi liquid [4]. Many impurity QCP have been predicted in exactly solvable one-dimensional correlated systems [5,6]. Renormalization group studies indicate that quantum impurity models flow toward a low-temperature fixed point corresponding to conformally invariant boundary conditions [7]. Also Wilson's numerical renormalization group maps the three-dimensional Kondo model to a one-dimensional Fermi lattice with a boundary impurity [8]. An impurity QCP is then given by the parafermionic sector of a Wess-Zumino-Novikov-Witten model.

Another topic of recent interest is the Kondo screening in Fermi systems with pseudo-gap, i.e., in systems where the density of states $\rho(\epsilon)$ has a power-law dependence on the energy $\epsilon$ near the Fermi level, $\rho(\epsilon) \sim \epsilon^{r}$. Using a renormalization-group (RG) analysis, Withoff and Fradkin [9] showed that there could be a critical value $J_{c}$ for the Kondo coupling constant, so that for $J>J_{c}$ Kondo screening occurs at low temperatures, while for $J<J_{c}$ the impurity decouples from the host and its magnetic susceptibility is divergent at $T=0$. Here the host itself is not a regular Fermi liquid and its low-temperature specific heat and susceptibility behave as $C \sim T^{r+1}$ and $\chi \sim T^{r}$, respectively. As discussed recently [10], the critical behavior of the impurity strongly depends on the host properties and appears to be non-universal. Due to the crossover regime from Kondo compensated to unscreened impurity spin almost all perturbation techniques fail in the critical regime. Under these circumstances, exact results, even for a special situation, may shed light onto this complicated subject.

In this letter, we study the Coqblin-Schrieffer impurity model in a correlated singular metal with $\rho(\epsilon) \sim \epsilon^{-1 / 2}$. In this case, the density of states at the Fermi level is divergent rather than zero. Such a situation can be realized when the Fermi level falls at the one-dimensional van Hove singularity of the electron band. The Hamiltonian is given by

$$
\begin{aligned}
H & =-\sum_{j=1}^{L-1} \sum_{s=-S}^{S} \mathcal{P}\left(c_{j, s}^{\dagger} c_{j+1, s}+\text { h.c. }\right) \mathcal{P}+\sum_{j=1}^{L-1} \sum_{s, s^{\prime}=-S}^{S}\left(c_{j, s}^{\dagger} c_{j, s^{\prime}} c_{j+1, s^{\prime}}^{\dagger} c_{j+1, s}+n_{j} n_{j+1}\right) \\
& +J \sum_{s, s^{\prime}=-S}^{S} c_{1, s}^{\dagger} c_{1, s^{\prime}} f_{s^{\prime}}^{\dagger} f_{s}+V n_{1}-\mu \sum_{j=1}^{L} n_{j},
\end{aligned}
$$

where $c_{j, s}^{\dagger}\left(c_{j, s}\right)$ is the creation (annihilation) operator of an electron at the site $j$ with spin component $s,-S \leq s \leq S$, $n_{j} \equiv \sum_{s} n_{j, s}=\sum_{s} c_{j, s}^{\dagger} c_{j, s}$ is the particle number operator, and $\mathcal{P} \cdots \mathcal{P}$ excludes the multiple occupation of each site $n_{j} \leq 1$. Here $J$ is the Kondo coupling constant, $V$ is a boundary potential, $\mu$ is the chemical potential, $f_{s}^{\dagger}\left(f_{s}\right)$ is the creation (annihilation) operator of the impurity with $\sum_{s} f_{s}^{\dagger} f_{s}=1$, so that there is always exactly one electron 
localized at the impurity. The Hamiltonian (1) describes a one-dimensional SU(N)-invariant $t-J$ model $(N=2 S+2)$ with a boundary impurity. Related $t-J$ models embedding a Coqblin-Schrieffer or Anderson impurity have been studied in Ref. 11].

We consider model (1) coupled to a reservoir of electrons, constituted by bands without direct interaction with the impurity. In this way the number of carriers in the chain changes with temperature if the chemical potential is fixed. At $\mu_{c}=-2$ the ground state of the $t-J$ model undergoes an insulator-metal transition from insulating for $\mu<\mu_{c}$ to a low carrier density conductor for $\mu>\mu_{c}$. At this critical point, the chain is a singular metal in the sense that the density of states for low-lying excitations diverges as $\rho(\epsilon) \sim \epsilon^{-1 / 2}$, which is due to the van Hove singularity.

The Hamiltonian (1) is exactly solvable via Bethe's Ansatz as a function of one parameter $v$ determining $J$ and $V$ via $J=\left(1-v^{2}\right)^{-1}, V=1+v /\left(1-v^{2}\right)$. For this parametrization the reflection matrix $R(q)$ of the electrons off the impurity satisfies the reflection Yang-Baxter equation (YBE)

$$
S\left(q_{i}-q_{j}\right) R\left(q_{i}\right) S\left(q_{i}+q_{j}\right) R\left(q_{j}\right)=R\left(q_{j}\right) S\left(q_{i}+q_{j}\right) R\left(q_{i}\right) S\left(q_{i}-q_{j}\right),
$$

where $S\left(q_{i} \pm q_{j}\right)$ denotes the two-body scattering matrix between electrons in the bulk [12]. Note that $v$ is required to be real to ensure a Hermitian Hamiltonian. Following the standard method outlined by Sklyanin [13] and its generalizations [14 we obtain the Bethe ansatz equations (BAE) diagonalizing the model in terms of $2 S+1$ sets of rapidities $\left\{q_{j}^{(r)}\right\}, r=0, \cdots, 2 S$,

$$
\begin{aligned}
& \left(\frac{q_{j}^{(0)}-\frac{i}{2}}{q_{j}^{(0)}+\frac{i}{2}}\right)^{2 L+1}=-\frac{q_{j}^{(0)}-i\left(\frac{3}{2}-v\right)}{q_{j}^{(0)}+i\left(\frac{3}{2}-v\right)} \prod_{p= \pm} \prod_{l \neq j}^{M_{0}} \frac{q_{j}^{(0)}-p q_{l}^{(0)}-i}{q_{j}^{(0)}-p q_{l}^{(0)}+i} \prod_{\alpha=1}^{M_{1}} \frac{q_{j}^{(0)}-p q_{\alpha}^{(1)}+\frac{i}{2}}{q_{j}^{(0)}-p q_{\alpha}^{(1)}-\frac{i}{2}}, \\
& \prod_{p= \pm} \prod_{t=0,2} \prod_{j=1}^{M_{t}} \frac{q_{\alpha}^{(1)}-p q_{j}^{(t)}-\frac{i}{2}}{q_{\alpha}^{(1)}-p q_{j}^{(t)}+\frac{i}{2}}=\frac{q_{\alpha}^{(1)}+i v}{q_{\alpha}^{(1)}-i v} \frac{q_{\alpha}^{(1)}+i(1-v)}{q_{\alpha}^{(1)}-i(1-v)} \prod_{p= \pm} \prod_{\beta \neq \alpha} \frac{q_{\alpha}^{(1)}-p q_{\beta}^{(1)}-i}{q_{\alpha}^{(1)}-p q_{\beta}^{(1)}+i}, \\
& \prod_{p= \pm} \prod_{t=r \pm 1} \prod_{l=1}^{M_{t}} \frac{q_{j}^{(r)}-p q_{l}^{(t)}-\frac{i}{2}}{q_{j}^{(r)}-p q_{l}^{(t)}+\frac{i}{2}}=\prod_{p= \pm} \prod_{m \neq j}^{M_{r}} \frac{q_{j}^{(r)}-p q_{m}^{(r)}-i}{q_{j}^{(r)}-p q_{m}^{(r)}+i}, \quad r>1
\end{aligned}
$$

where $M_{r}=\sum_{l=r}^{2 S} n_{l-S}(r=0, \cdots, 2 S)$ and $M_{2 S+1}=0$. The impurity gives rise to the factors involving $v$ in the first and second set of equations (3). The set of rapidities $\left\{q_{j}^{(0)}\right\}$ refers to the charges, while the remaining sets for $r=1, \cdots, 2 S$ correspond to the spin degrees of freedom. The energy of the system is given by

$$
E=\sum_{j=1}^{M_{0}}\left[2-\frac{1}{\left(q_{j}^{(0)}\right)^{2}+\frac{1}{4}}-\mu\right] .
$$

We first briefly summarize the properties of the chain without the impurity. In the thermodynamic limit the solutions of the BAE are given in terms of string excitations of arbitrary length $n$ of the form $q^{(r)}=\Lambda+i(n-1-2 l) / 2$ with $l=0, \cdots, n-1$ for each of the sets of rapidities. Here $\Lambda$ represents the rapidity of the center of mass of the string. We denote with $\varphi_{n}^{(r)}(\Lambda)=T \ln \left[\eta_{r, n}(\Lambda)\right]$ the dressed energy of the corresponding string excitations, which satisfy the following integral equations obtained by minimizing the free energy 15]

$$
\begin{aligned}
\ln \left(1+\eta_{r, n}(\Lambda)\right) & =\frac{\epsilon_{r, n}(\Lambda)}{T}+\sum_{m=1}^{\infty} A_{n m} \star \ln \left(1+\eta_{r, m}^{-1}\right) \\
& -\sum_{m=1}^{\infty} B_{n m} \star\left[\ln \left(1+\eta_{r-1, m}^{-1}\right)+\ln \left(1+\eta_{r+1, m}^{-1}\right)\right]
\end{aligned}
$$

where $T$ is the temperature and $\star$ represents a convolution. Here $\epsilon_{0, n}(\Lambda)=n(2-S h-\mu)-2 \pi a_{n}(\Lambda), \epsilon_{r, n}=n h$ for $r=$ $1, \cdots, 2 S$, and $h$ is the magnetic field. The integration kernels are $A_{n m}=a_{m+n}+a_{|m-n|}+2 \sum_{l=1}^{\min (m, n)-1} a_{m+n-2 l}$ and $B_{m n}=\sum_{l=1}^{\min (m, n)} a_{m+n-2 l+1}$, where $a_{n}(\Lambda)=|n| /\left[2 \pi\left(\Lambda^{2}+n^{2} / 4\right)\right]$. In Eqs. (5) we assumed that $\eta_{2 S+1, n}=\eta_{-1, n}=\infty$ for all $n$. The free energy of the host is given by $F=L f_{\text {host }}$, where

$$
f_{\text {host }}=-T \sum_{n} \int d \Lambda a_{n}(\Lambda) \ln \left[1+\eta_{0, n}^{-1}(\Lambda)\right] .
$$


In the ground state all the rapidities are real, i.e. only states with $n=1$ can be occupied. From the expression of the energy it is seen that in zero-field for $\mu \leq \mu_{c}=-2$ the band of charge rapidities $(r=0)$ is empty and the system is an insulator. For $\mu$ slightly larger than $\mu_{c}$ the system has a low-density of carriers. The dressed energy for the charges for this case is given by

$$
\varphi_{1}^{(0)}(\Lambda)=2-S h-\mu-2 \pi a_{1}(\Lambda) \approx-S h-\mu-2+16 \Lambda^{2},
$$

where in the last step we assumed $|\Lambda|$ small. The Fermi points are determined by $\varphi_{1}^{(0)}( \pm Q)=0$, i.e. $Q=$ $\frac{1}{4} \sqrt{2+\mu+S h}$, which in zero-field tends to zero at $\mu_{c}$. The ground state energy and susceptibility for small $Q$ are then given by

$$
E_{\text {host }} / L=-\frac{2}{3 \pi}(S h+\mu+2)^{3 / 2} \quad, \quad \chi_{\text {host }} / L=\left(S^{2} / 2 \pi\right)(S h+\mu+2)^{-1 / 2} .
$$

The expression for $\chi_{\text {host }}$ diverges with a square root singularity as a consequence of the one-dimensional van Hove singularity of the empty charge rapidity band.

Similarly, the low-temperature thermodynamics of the bulk of the chain can be derived to leading order. The main contribution to the free energy arises from $\varphi_{1}^{(0)}$; substituting Eq. (7) into Eq. (6), we obtain

$$
f_{\text {host }}=-c_{1} T^{\frac{3}{2}}-c_{2} T^{-\frac{1}{2}} h^{2}+\cdots,
$$

where $c_{1}=\int \ln \left(1+e^{-x^{2}}\right) d x$ and $c_{2}=\frac{1}{8} S^{2} \int \cosh ^{-2}\left(x^{2} / 2\right) d x$. The specific heat and the susceptibility are then

$$
C_{\text {host }} / L=\frac{3}{4} c_{1} T^{\frac{1}{2}}+\cdots \quad, \quad \chi_{\text {host }} / L=2 c_{2} T^{-\frac{1}{2}}+\cdots .
$$

This result indicates that a non-interacting charge model already gives exactly the leading order of the low-temperature thermodynamics. Because of the low carrier density the interactions between particles can plausibly be omitted, since they contribute only to subleading order.

In general the impurity can give rise to imaginary modes, which correspond to boundary bound states involving impurity states. From the first set of the BAE, Eqs. (3), we see that $q^{(0)}=i(3 / 2-v)$ for $v<3 / 2$ is a solution in the thermodynamic limit $L \rightarrow \infty$. The energy associated with this bound state is

$$
\epsilon_{b}=2-\mu+\frac{1}{(v-1)(v-2)},
$$

so that it is stable only in a small interval of $v$ for $v>1$. This corresponds to ferromagnetic coupling of the impurity to the chain. In the antiferromagnetic Kondo coupling regime $J>0(|v|<1)$, the energy of this state is a high energy excited charge bound state.

A spin-charge bound state should exist, because when $v \rightarrow-1^{+}$then $J \rightarrow+\infty$ and $V \rightarrow-\infty$, i.e. both the Kondo coupling and the attractive boundary potential enforce the formation of a stable boundary bound state between the impurity and one bulk particle. A detailed analysis of the second set of BAE shows that $q^{(1)}=i v$ is a solution if $q^{(0)}=i(v+1 / 2)$ is a solution of the first set of Eqs. (3). This is indeed the case in the interval $-1<v<-1 / 2$, where this state describes the spin singlet pairing of the impurity with one electron of the bulk. Interestingly, this bound state is stable even in the insulating phase $\left(\mu<\mu_{c}\right)$, as long as $\mu \geq 2+1 /\left(v+v^{2}\right)$, i.e., the Kondo screening exist if the Kondo coupling is strong enough to overcome the energy gap of the charges.

Below we examine how the impurity behaves in the neighborhood of the quantum critical point $\mu_{c}=-2$. Two situations have to be distinguished in the ground state, namely, for $-1<v<-1 / 2$, the local moment of the impurity is quenched into a singlet by one host electron, while for $-1 / 2 \leq v<1$, the ground state is $(2 S+1)$-fold degenerate and an arbitrary small magnetic field induces a finite magnetization $S$. In other words, there is a first order phase transition at $v=-1 / 2$. Such a critical point is indeed very similar to the $J_{c}$ found in Ref. [9].

The free energy of the impurity is driven by the host via the functions $\eta_{r, n}(\Lambda)$,

$$
f_{i m p}=-T \sum_{r=0,1} \sum_{n} \int d \Lambda \phi_{r, n}(\Lambda) \ln \left[1+\eta_{r, n}^{-1}(\Lambda)\right]
$$

with 


$$
\begin{aligned}
\phi_{0, n}(\Lambda) & =\frac{1}{2} \sum_{l=1}^{n} a_{|n-2 l+2 v-2|}(\Lambda) \operatorname{sign}(n-2 l+2 v-2), \\
\phi_{1, n}(\Lambda) & =\frac{1}{2} \sum_{l=1}^{n} a_{|n-2 l+2 v+1|}(\Lambda) \operatorname{sign}(n-2 l+2 v+1) \\
& +\frac{1}{2} \sum_{l=1}^{n} a_{|n-2 l-2 v+3|}(\Lambda) \operatorname{sign}(n-2 l-2 v+3)
\end{aligned}
$$

for $-\frac{1}{2} \leq v<1$, and

$$
\begin{aligned}
\phi_{0, n}(\Lambda) & =\frac{1}{2} \sum_{l=1}^{n} a_{|n-2 l+2 v+2|}(\Lambda) \operatorname{sign}(n-2 l+2 v+2) \\
& +\frac{1}{2} \sum_{l=1}^{n} a_{|n-2 l+2 v-2|}(\Lambda) \operatorname{sign}(n-2 l+2 v-2) \\
& +\frac{1}{2} \sum_{l=1}^{n} a_{|n-2 l-2 v-2|}(\Lambda) \operatorname{sign}(n-2 l-2 v-2) \\
\phi_{1, n}(\Lambda) & =0
\end{aligned}
$$

for $-1<v<-\frac{1}{2}$. Here we have omitted the unstable charge boundary bound state and the unstable charge-spin bound state for $-1 / 2 \leq v<1$, as well as the excitations arising from them, since their contributions are exponentially small at sufficiently low temperatures. In Eq. (12) we also omitted the energy of the stable bound state for $-1<v<-\frac{1}{2}$, which is just an additive temperature and field independent constant, $\epsilon_{b s}=2-\mu+1 /[v(v+1)]$.

In the ground state only the $n=1$ strings are relevant. Using Eq. (7) and following arguments similar to those used to derive Eq. (8), we obtain

$$
\begin{aligned}
& \chi_{i m p}=S \delta(h)-\frac{S^{3 / 2}}{4 \pi} \frac{1}{(3-2 v)} h^{-\frac{1}{2}}+\cdots, \quad \text { for } \quad-\frac{1}{2} \leq v<1, \\
& \chi_{i m p}=-\frac{S^{3 / 2}}{4 \pi} \frac{\left(4 v^{2}+12 v-3\right)}{\left(9-4 v^{2}\right)(2 v+1)} h^{-\frac{1}{2}}+\cdots, \quad \text { for } \quad-1<v<-\frac{1}{2} .
\end{aligned}
$$

The second term for $-1 / 2 \leq v<1$ is negative, which means that the local moment is not completely free as a consequence of the Kondo coupling of the impurity to the bulk modes, which yield contributions to subleading order. Note that for $-1<v<-\frac{1}{2} \chi_{i m p}$ is negative because one spin from the host is locked into the singlet boundary bound state. From Eq. (8) we have that the host susceptibility is also proportional to $h^{-\frac{1}{2}}$, so that as expected the critical behavior of the impurity is locked into that of the host.

At low temperatures the charge strings for $n>1$ are gapped and for $\mu \approx \mu_{c}$ we have

$$
\eta_{0, n}(\Lambda) \approx \exp \left[\frac{n(2-\mu-S h)-2 \pi a_{n}(\Lambda)}{T}\right] .
$$

Hence, their contributions to the free energy are exponentially small and can be neglected, except for $\eta_{0,1}(\Lambda)$ which may have a Fermi surface and contributes to leading order to the low- $T$ thermodynamics. In addition, at low $T$ and for $\mu \approx \mu_{c}$ the spin and charge degrees of freedom are only weakly coupled. Hence, all $\eta_{r, n}(\Lambda)$ for $r \geq 1$ tend to constants, so that the integral equations determining the $\eta_{r, n}$ reduce to algebraic ones and are easily solved [16, 18]

$$
\eta_{r, n}+1=\frac{\sinh \left[(n+r) x_{0}\right] \sinh \left[(n+2 S-r+1) x_{0}\right]}{\sinh \left(r x_{0}\right) \sinh \left[(2 S+1-r) x_{0}\right]}
$$

where $x_{0}=h / 2 T$.

For the low-temperature thermodynamics of the impurity, we distinguish three cases: (i) $0<v<1$, (ii) $-1 / 2 \leq$ $v \leq 0$, and (iii) $-1<v<-1 / 2$. For case (i) the free energy of the impurity can be approximated by

$$
f_{i m p} \approx \frac{1}{2} T \int d \Lambda a_{3-2 v}(\Lambda) \ln \left[1+\eta_{0,1}^{-1}(\Lambda)\right]-S h-T \sum_{n=1}^{\infty} \ln \left(1+\eta_{1, n}^{-1}\right) .
$$


Using Eq. (17) the last two terms can be rewritten as $-T \ln \left\{\sinh \left[(2 S+1) x_{0}\right] / \sinh \left(x_{0}\right)\right\}$, which is just the free energy of the free spin. This clearly indicates that in the region (i) the charge and spin degrees of freedom are decoupled. The specific heat of the charges is related to that of the bulk by $C_{i m p}=[1 /(4 v-6)] C_{b u l k} \sim T^{\frac{1}{2}}$ (as in Eq. (15) this contribution is negative), while the spin part gives rise to a Schottky anomaly. The zero-field residual entropy $S_{\text {res }}(T \rightarrow 0)$ is $\ln (2 S+1)$, which exactly corresponds to a free spin $S$. The impurity susceptibility follows a Curie law with a subleading term $[1 /(4 v-3)] \chi_{\text {bulk }} \sim T^{-\frac{1}{2}}$, which arises from the charges.

For case (ii), $-1 / 2 \leq v<0$, again the charge and spin degrees of freedom decouple. While the charge part is identical to case (i) (and so is the $T^{\frac{1}{2}}$ dependence of the specific heat and the $T^{-\frac{1}{2}}$ dependence of the susceptibility), the spin contribution to the impurity free energy is now $-S h-T \sum_{n=2}^{\infty} \ln \left(1+\eta_{1, n}^{-1}\right)=-T \ln \left\{\sinh \left[(2 S+2) x_{0}\right] / \sinh \left(2 x_{0}\right)\right\}$. Consequently, the Schottky anomaly and the Curie constant are changed to that of an effective spin that in general is neither integer nor half-integer. The residual zero-field entropy at finite but low $T$ is $\operatorname{then} S_{\text {res }}=\ln (S+1)$, i.e. it is reduced by the Kondo coupling with respect to the free spin. Hence, as a function of $v$ the entropy has a jump at $v=0$ of

$$
\Delta S_{\text {res }}=\ln \left[\frac{2 S+1}{S+1}\right] .
$$

The reduction of the entropy indicates that partial Kondo screening occurs at low but finite temperatures. However, the degeneracy of the ground state in zero-field is exactly $(2 S+1)$-fold and the entropy is continuous as a function of $T$.

This unusual phenomenon can be understood within the following picture. In the ground state there are no excited states to screen the impurity and thus there is no Kondo effect. However, at finite temperature, there exist bulk excitations which partially screen the local moment. It is this activated Kondo effect that induces a discontinuity in the entropy as a function of $v$ at $v=0$. The situation encountered here is very different from the one in a Fermi liquid or in a Luttinger liquid, where there are sufficient conduction electrons to compensate the impurity spin at any temperature. Hence, the lower $T$, the stronger the Kondo screening. In the present case, however, $T$ itself controls the number of carriers and therefore the Kondo compensation. Experimentally, the activated Kondo effect could occur in dilute magnetically doped narrow-gap semiconductors, where thermally activated carriers may induce a finite temperature Kondo effect. For $v>0$, the larger boundary potential $V$ dominates over the Kondo coupling $J$ and repels the bulk particles from the impurity. This explains the absence of a temperature activated Kondo effect in this parameter region.

Finally for $-1<v<-1 / 2$, case (iii), we have from Eq. (14) that at very low temperature the impurity only contributes to the free energy in the charge sector because $\phi_{1, n}=0$. We obtain for this case

$$
\begin{aligned}
C_{i m p} & =\frac{\left(-4 v^{2}-12 v+3\right)}{\left(9-4 v^{2}\right)(2 v+1)} C_{\text {host }}, \\
\chi_{i m p} & =\frac{\left(-4 v^{2}-12 v+3\right)}{\left(9-4 v^{2}\right)(2 v+1)} \chi_{h o s t}, \\
S_{r e s}(0) & =S_{\text {res }}(T \rightarrow 0)=0,
\end{aligned}
$$

which means the critical behavior of the impurity is locked into that of the bulk, as in the case of Fermi liquids.

In conclusion, we have studied the properties of a Coqblin-Schrieffer impurity in a singular metal. At low temperatures three situations have to be distinguished, namely, the standard Kondo screening of the impurity into a singlet state, an "activated Kondo effect" in which a partial spin compensation is induced via thermal activation, and for repulsive coupling at the boundary the impurity spin is asymptotically free. As predicted in Ref. [9] for a semi-metal a critical value for the coupling constant is required, below which the zero temperature Kondo screening disappears. We suggest that a possible realization of the thermally "activated Kondo effect" could be in very-narrow-gap semiconductors.

We acknowledge the support by the National Science Foundation and the Department of Energy under grants No. DMR98-01751 and No. DE-FG02-98ER45797. Y. Wang is also supported by the National Science Foundation of China. 
[1] M.B. Maple et al., J. Low Temp. Phys. 99, 223 (1995); H. von Löhneysen et al., Physica (Amsterdam) B 230-232, 550 (1997).

[2] P. Noziéres and A. Blandin, J. Phys. (Paris) 41, 193 (1980).

[3] N. Andrei and C. Destri, Phys. Rev. Lett. 52, 364 (1984); P.B. Wiegmann and A.M. Tsvelik, JETP Lett. 38, 596 (1983); P. Schlottmann and P.D. Sacramento, Adv. Phys. 42, 441 (1993).

[4] D.-H. Lee and J. Toner, Phys. Rev. Lett. 69, 3378 (1992); A. Furusaki and N. Nagaosa, Phys. Rev. Lett. 72, 892 (1994); P. Fröjdh and H. Johannesson, Phys. Rev. Lett. 75, 300 (1995).

[5] Y. Wang et al., Phys. Rev. Lett. 79, 1901 (1997); Y. Wang, Phys. Rev. B 56, 14045 (1997); J. Dai, Y. Wang and U. Eckern, Phys. Rev. B 60, 6594 (1999).

[6] A. Zvyagin and H. Johannesson, Phys. Rev. Lett. 81, 2751 (1998); A. Zvyagin and P. Schlottmann, J. Phys. A 31, 1981 (1998); Nucl. Phys. B 565 [FS], 555 (2000).

[7] A.W.W. Ludwig and I. Affleck, Nucl. Phys. B 428 [FS], 545 (1994); I. Affleck in Springer Series in Solid State Sciences, 118, ed. by A. Okiji and N. Kawakami (Springer-Verlag, 1994) and references therein.

[8] K.G. Wilson, Rev. Mod. Phys. 47, 773 (1975).

[9] D. Withoff and E. Fradkin, Phys. Rev. Lett. 64, 1835 (1990).

[10] C.R. Cassanello and E. Fradkin, Phys. Rev. B 53, 15079 (1996); 56, 11246 (1997); K. Ingersent, Phys. Rev. B 54, 11936 (1996); C. Gonzalez-Buxton and K. Ingersent, Phys. Rev. B 57, 14254 (1998).

[11] P. Schlottmann, J. Phys.: Cond. Matter 10, 2525 (1998); P. Schlottmann and A. Zvyagin, Euro. Phys. J. B 5, 325 (1998).

[12] B. Sutherland, Phys. Rev. B 12, 3795 (1975); P. Schlottmann, Phys. Rev. B 36, 5177 (1987).

[13] E.K. Sklyanin, J. Phys. A 21, 2375 (1988).

[14] A. Foerster and M. Karowski, Nucl. Phys. B 396, 11 (1993); C. Destri and H.J. de Vega, Nucl. Phys. B 361, 361 (1992); ibid 374, 692 (1992).

[15] M. Takahashi, Prog. Theor. Phys. 46, 401 (1971).

[16] N. Andrei, K. Furuya and J.H. Lowenstein, Rev. Mod. Phys. 55, 331 (1983).

[17] H. Johannesson, Phys. Lett. A 116, 133 (1986).

[18] P. Schlottmann, Phys. Rep. 181, 1 (1989). 